\title{
Three-dimensional rotational angiography in diagnosis of chronic thromboembolic pulmonary hypertension
}

\author{
Michał A. Potępa ${ }^{1}$, Andrzej Łabyk ${ }^{1}$, Dariusz Zieliński², Piotr Pruszczyk ${ }^{1}$, Marek Roik ${ }^{1}$ \\ ${ }^{1}$ Department of Internal Medicine and Cardiology with the Centre for Management of Venous Thromboembolic Disease, Medical University \\ of Warsaw, Warsaw, Poland \\ ${ }^{2}$ Cardiac Surgery Department, Medicover Hospital, Warsaw, Poland
}

Adv Interv Cardiol 2021; 17, 3 (65): 327-329

DOI: https://doi.org/10.5114/aic.2021.109165

Chronic thromboembolic pulmonary hypertension (CTEPH) is a rare disease resulting from stenosis or obstruction of the pulmonary arteries due to organized thrombus with disruption of blood flow and derivative microvascular remodelling in $4 \%$ of survivors of acute pulmonary embolism (PE). Computed tomography (CT) angiography and conventional pulmonary angiography remain the gold standard to confirm the presence of fibrotic thromboembolic lesions in pulmonary arteries [1, 2]. Pulmonary endarterectomy (PEA) remains the treatment of choice for $\mathrm{CTEPH}$, but in recent years, there has been significant progress in the development of alternate treatments for patients deemed non-operable such as balloon pulmonary angioplasty (BPA) or targeted drug therapy. Patient eligibility for PEA or BPA requires multidisciplinary evaluation at a specialized centre [1-4]. However, interpretation difficulties exist for CT imaging, especially at the level of segmental and subsegmental arteries. This is crucial in deeming eligibility for surgery versus percutaneous intervention. Here, we present a case of a 69-year-old man admitted with a high likelihood of CTEPH. The patient did not have significant comorbidities and reported gradual deterioration of exercise tolerance and exertional dyspnoea (World Health Organization functional class II) since having an acute pulmonary embolism episode in 2019, despite receiving long-term warfarin anticoagulant therapy. Echocardiography performed 6 months after the incident revealed signs of right ventricular dysfunction with high suspicion of pulmonary hypertension (tricuspid regurgitation pressure gradient (TRPG) $76 \mathrm{~mm} \mathrm{Hg}$, right ventricular systolic pressure (RVSP) $81 \mathrm{~mm} \mathrm{Hg}$, tricuspid annular plane systolic excursion (TAPSE) 17, with preserved left ventricle ejection fraction of $60 \%$ ). Pulmonary single photon emission computed tomography (SPECT) performed using albumin macroaggregates labelled with radioactive technetium showed multiple bilateral, diffuse segmental perfusion defects typical of CTEPH. The patient underwent right heart catheterization (RHC) using the transjugular approach, which confirmed elevated mean pulmonary artery pressure (mPAP - $40 \mathrm{~mm} \mathrm{Hg}$ ), normal right arterial pressure $(2 \mathrm{~mm} \mathrm{Hg})$ and pulmonary wedge pressure (4 $\mathrm{mm} \mathrm{Hg}$ ), with a calculated pulmonary vascular resistance (PVR) of 7.55 Wood units. Rotational pulmonary angiography (with $50 \mathrm{ml}$ of contrast medium administered over $5 \mathrm{~s}$ of continuous infusion; $6 \mathrm{~F}$ pigtail catheter) was performed simultaneously and showed lesions typical of CTEPH (ring-like stenosis, pouches, intravascular webs, subtotal occlusions) in the segmental and sub-segmental arteries of both lungs. Due to the complexity of the anatomy of the pulmonary arteries in patients with CTEPH (Figures $1 \mathrm{~A}, \mathrm{C}$ ), we performed three-dimensional rotational reconstruction with post-processing (Figures $1 \mathrm{~B}, \mathrm{D}$ ) to achieve optimal visualization in the characterization of the lesions. According to current guidelines, to determine operability (PEA), the patient had a consultation with an experienced cardiac surgeon and was qualified for PEA. Thus, the patient underwent uneventful PEA with marked improvement of hemodynamic parameters (mPAP 25 mm Hg, PVR 3.5 Wood units) (Figure 2).

In summary, three-dimensional rotational angiography reconstruction can be a reasonable tool in the diagnostic work-up of CTEPH and for planning interventional or surgical treatment. Detailed reconstruction of the pulmonary arteries allows for the selection of appropriate balloon catheters that are even appropriate for distally located lesions. It can also provide additional information for PEA. Nevertheless, this strategy needs further assessment in a larger group of CTEPH patients.

\section{Corresponding author:}

Michał A. Potępa MSc, Department of Internal Medicine and Cardiology with the Centre for Management of Venous Thromboembolic Disease, Medical University of Warsaw, Warsaw, Poland, e-mail: m.a.potepa@gmail.com

Received: 15.07.2021, accepted: 16.08.2021. 

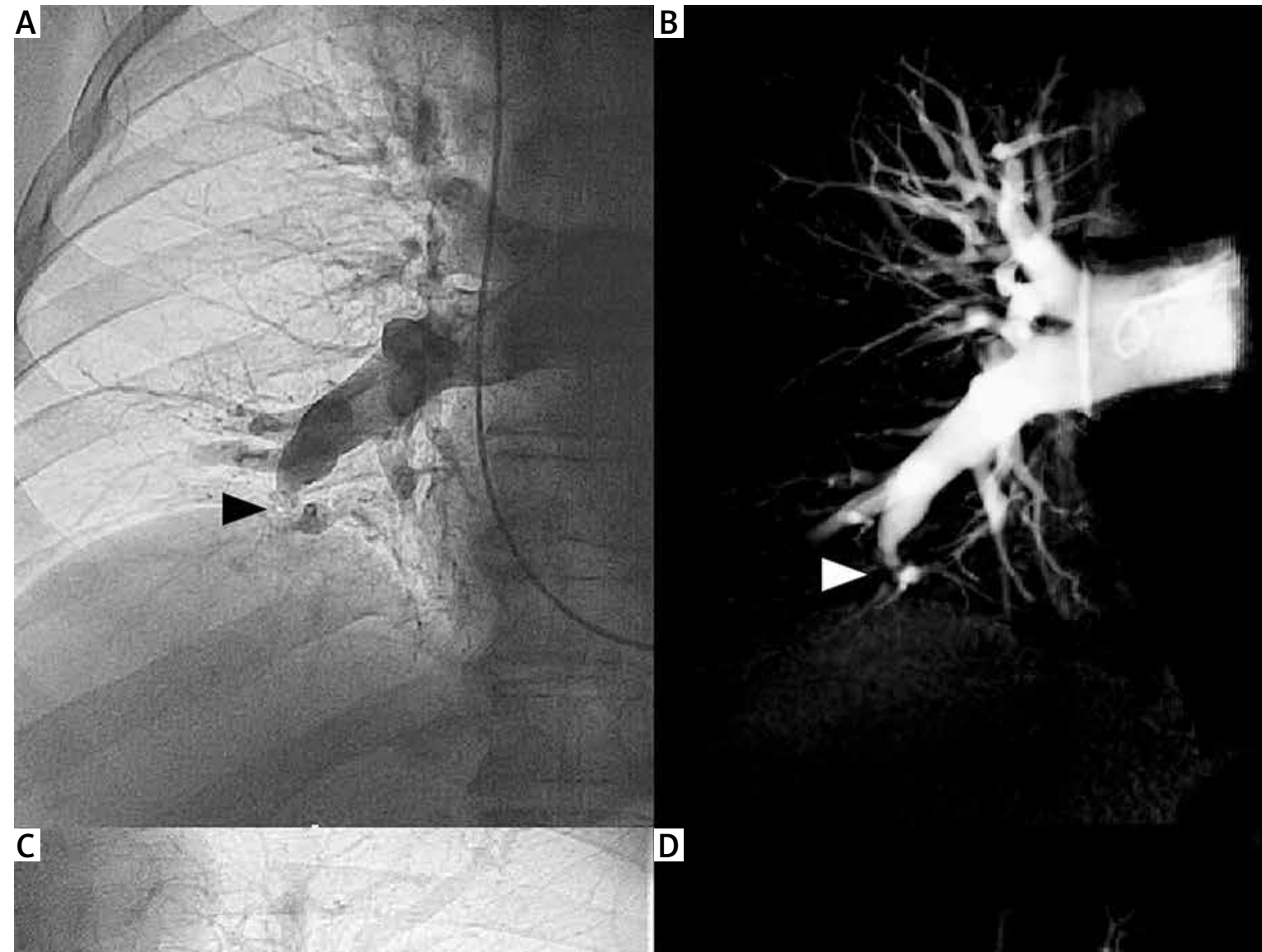

D

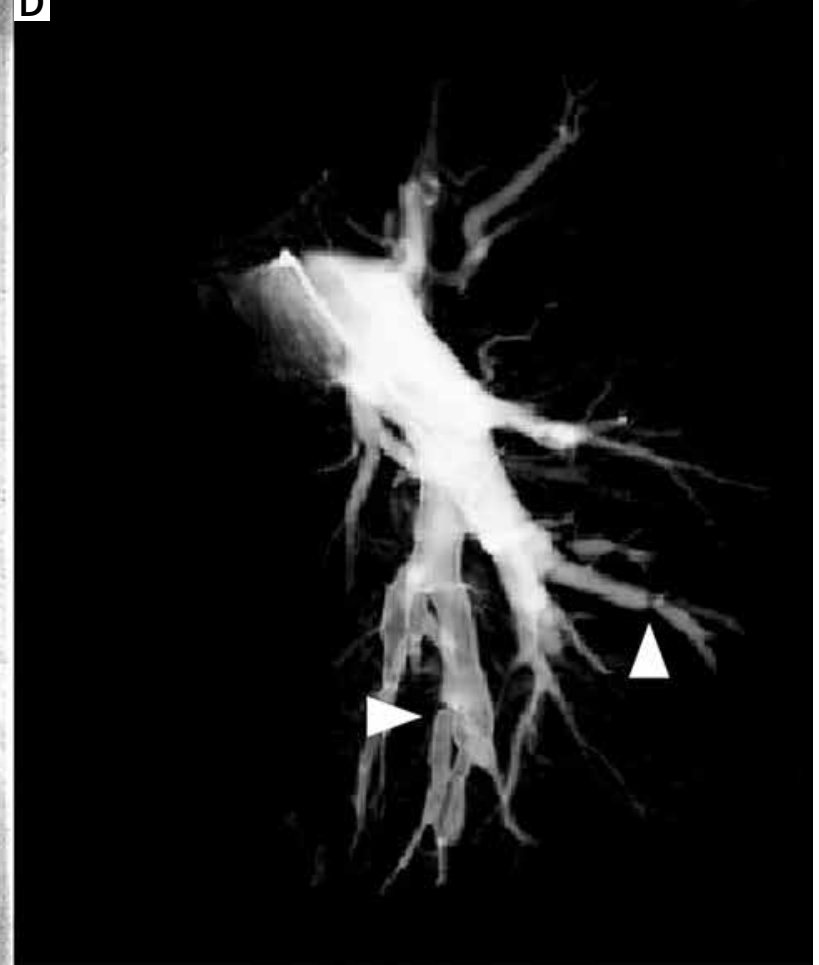

Figure 1. A - Right pulmonary artery angiography, AP-view; lower lobe pulmonary artery occlusion - pouch lesion (arrow). B - Three-dimensional rotational angiography reconstruction of right pulmonary artery, AP-view; lower lobe pulmonary artery occlusion - pouch (arrow). C - Left pulmonary artery angiography, AP-view; ringlike stenosis (arrow). D - Three-dimensional rotational angiography reconstruction of left pulmonary artery, AP-view; ring-like stenosis (arrow) 

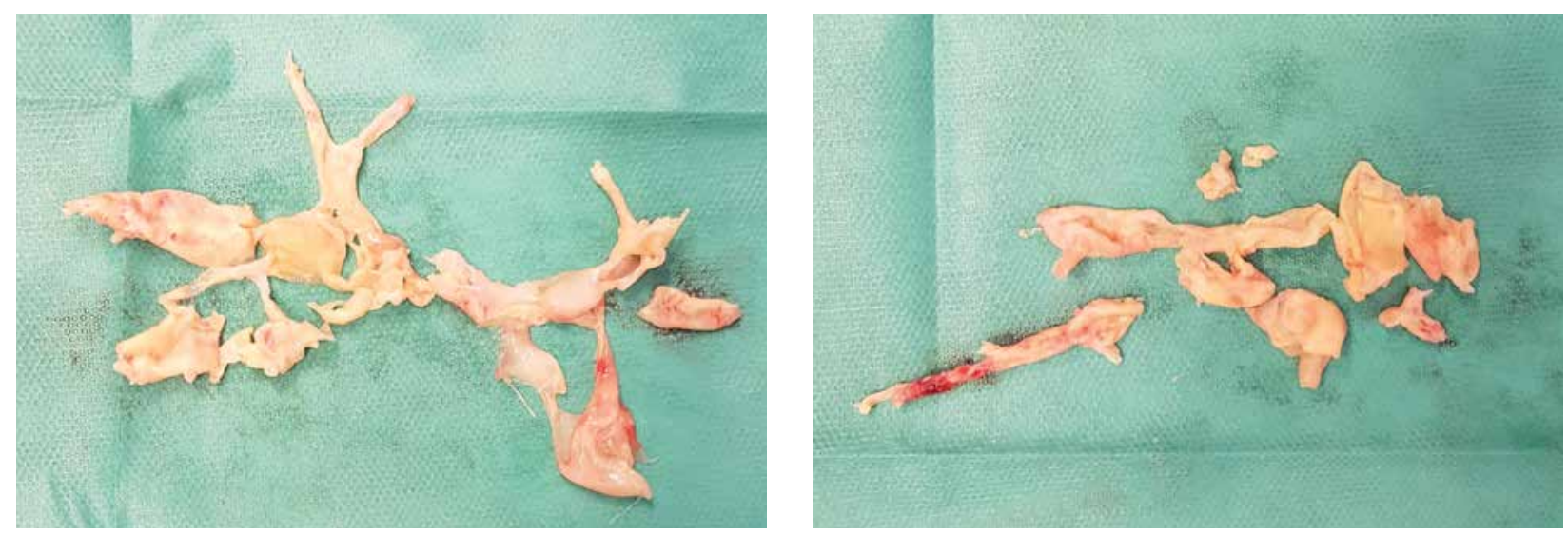

Figure 2. Surgical specimen resected during successful pulmonary endarterectomy

\section{Conflict of interest}

The authors declare no conflict of interest.

\section{References}

1. Konstatinids SV, Meyer G, Becattini C, et al. 2019 ESC Guidelines for the diagnosis and management of acute pulmonary embolism developed in collaboration with the European Respiratory Society (ERS): The Task Force for the diagnosis and management of acute pulmonary embolism of the European Society of Cardiology (ESC). Eur Heart J 2020; 41: 543-603.

2. Auger W. Surgical and percutaneous interventions for chronic thromboembolic pulmonary hypertension. Cardiol Clin 2020; 38: 257-68.

3. Roik $M$, Wretowski $D$, tabyk $A$, et al. Refined balloon pulmonary angioplasty driven by combined assessment of intra-arterial anatomy and physiology--Multimodal approach to treated lesions in patients with non-operable distal chronic thromboembolic pulmonary hypertension--Technique, safety and efficacy of 50 consecutive angioplasties. Int J Cardiol 2016; 203: 228-35.

4. Roik M, Wretowski D, Kostrubiec M, et al. High prevalence of severe coronary artery disease in elderly patients with non-operable chronic thromboembolic pulmonary hypertension referred for balloon pulmonary angioplasty. Adv Interv Cardiol 2016; 12: 355-9. 